\title{
Crystal structures and small angle $x$-ray scattering analysis of antifungal drug target aspartate semialdehyde dehydrogenase
}

\author{
Gopal P. Dahal ${ }^{\mathrm{a}}$, Shuo Qian $^{\mathrm{b}}$ and Ronald E. Viola ${ }^{\mathrm{a}}$ \\ ${ }^{a}$ Department of Chemistry and Biochemistry, The University of Toledo, Toledo, Ohio 43606 \\ ${ }^{b}$ Biology and Soft Matter Division, Oak Ridge National Laboratory, Oak Ridge, Tennessee \\ 37831, United States
}

Aspartate semialdehyde dehydrogenase (ASADH) functions at a critical junction in the aspartate biosynthetic pathway and represents a validated target for antimicrobial drug design. This enzyme catalyzes the NADPH-dependent reductive dephosphorylation of $\beta$-aspartyl phosphate to produce the key intermediate aspartate semialdehyde. Production of this intermediate represents the first committed step for the biosynthesis of essential amino acids in fungi and in bacteria. The absence of this enzyme in humans and other mammals will allow selective targeting of pathogenic microorganisms. We have accumulated significant structural and mechanistic information about the bacterial ASADHs, but have only limited knowledge of their fungal counterparts. We have recently determined the high resolution structure of two new pathogenic fungal forms of ASADH from Cryptococcus neoformans ${ }^{l}$ and Aspergillus fumigatus ${ }^{2}$. While the overall structures are similar to the bacterial orthologs, some critical differences both in biological assembly and in secondary structural features can potentially be exploited for the development of species-selective drugs with selective toxicity against infectious fungal organisms. Native PAGE studies, small angle x-ray scattering (SAXS) analysis from the Guinier region and ab initio modelling from DAMMIF each show that the fungal ASADHs exist as a tetramer ${ }^{3}$. A customized fragment library has been screened against several fungal ASADHs and initial hits have been identified with both in vitro and cell-based inhibition values in the low micromolar range. Some of these potent inhibitors show a noncompetitive mode of inhibition, functioning to disrupt the tetrameric interface and cause dissociation into inactive dimers. Docking studies with these inhibitor structures are being used to guide the design and development of more potent inhibitors. The long term goal of this project is to develop drugs that selectively target these fungal enzymes and inhibit the growth of these pathogenic fungal species.

\section{References:}

1. Dahal, G.; Viola, R. E., Structure of a fungal form of aspartate semialdehyde dehydrogenase from Cryptococcus neoformans. Acta Crystallographica Section F 2015, 71 (11), 1365-1371.

2. Dahal, G.; Viola, R. E., Structure of a fungal form of aspartate-semialdehyde dehydrogenase from Aspergillus fumigatus. Acta Crystallographica Section F 2017, 73 (1), 3644.

3. Li, Q.; Mu, Z.; Zhao, R.; Dahal, G.; Viola, R. E.; Liu, T.; Jin, Q.; Cui, S., Structural Insights into the Tetrameric State of Aspartate- $\beta$-semialdehyde Dehydrogenases from Fungal Species. Scientific Reports 2016, 6, 21067. 